\title{
ALGUNAS PRECISIONES SOBRE LOS DELITOS DE ENRIQUECIMIENTO ILÍCITO Y LAVADO DE ACTIVOS
}

\author{
María del Carmen García Cantizano* \\ Universidad de Lima, Lima, Perú \\ Recibido: 17/11/2020 Aprobado: 1/12/2020 \\ doi: https://doi.org/10.26439/iusetpraxis2021.n053.4970
}

\begin{abstract}
RESUMEN. En este artículo se analizan los principales elementos que conforman la estructura típica del delito de enriquecimiento ilícito con el objetivo de delimitar en su justa medida cuál es el ámbito concreto de su aplicación legal en la práctica. A partir de ahí, se pretende definir qué relación existe entre los delitos de enriquecimiento ilícito y lavado de activos a fin de establecer criterios que faciliten una adecuada subsunción bajo cada uno de estos tipos penales.
\end{abstract}

PALABRAS CLAVE: enriquecimiento ilícito / lavado de activos / funcionario público / delito fuente / abuso del cargo / delito especial / complicidad / concurso de delitos

\section{SOME CLARIFICATIONS ON ILLICIT ENRICHMENT AND MONEY LAUNDERING OFFENSES}

ABSTRACT. The present work analyzes the main elements that make up the typical structure of the crime of illicit enrichment to delimit in its fair measure the specific scope of its legal application in practice. We intended to define the relationship between illicit enrichment and money laundering crimes to establish criteria that facilitate adequate subsumption under each of these criminal types.

KEYWORDS: illicit enrichment / money laundering / civil servant / source offense / abuse of office / special offense / complicity / crime contest

\footnotetext{
* Doctora en Derecho por la Universidad de Sevilla, España. Profesora de Derecho Penal de la Universidad de Lima y de la Pontificia Universidad Católica del Perú. Abogada del Estudio Bramont-Arias Torres.
} 


\section{INTRODUCCIÓN}

En tiempos especialmente convulsos, cuando los ciudadanos son testigos de comportamientos y actitudes absolutamente intolerables por parte de quienes los representan y, por ello, han asumido la defensa teórica de sus intereses, el recurso al derecho penal se presenta - a veces- como la única solución capaz de apaciguar la más que justificada crítica ciudadana frente al Estado. La corrupción implantada en el seno de la Administración Pública constituye un lastre muy pesado que llega a afectar incluso a su misma legitimidad: no hay más que revisar las encuestas públicas para apreciar la mala imagen que tienen de cara al ciudadano de a pie determinadas instituciones que son esenciales y básicas para el adecuado funcionamiento del Estado.

Ante semejante panorama, la respuesta del legislador suele consistir bien en el aumento de las penas para aquellos delitos que adquieren mayor notoriedad pública, o bien en la incorporación de circunstancias que permiten intensificar las sanciones, o en la incorporación de nuevas figuras delictivas que amplían el ámbito de acción del derecho penal, más allá de una necesaria justificación basada en lo que algunos seguimos considerando fundamental, esto es, la protección de un bien jurídico.

En este sentido, consideramos que tanto el delito de enriquecimiento ilícito como el de lavado de activos son, en cierta medida, un claro ejemplo de estas últimas tendencias en materia de política criminal, en las cuales la falta de eficacia en la lucha contra cierta clase de criminalidad, como la corrupción en el seno de la Administración Pública, pretende ser disipada sobre la base de tipos penales en los que no se sabe qué comportamiento se quiere realmente sancionar, ni queda del todo claro qué objeto ayudan a proteger. Con ello, lejos de asegurar la eficacia de la norma penal ya existente, se contribuye a generar serios problemas de interpretación entre tipos delictivos, lo que, a su vez, provoca conflictos entre las diferentes normas penales, por cuanto se superponen en su ámbito de aplicación. Esto hace necesario acudir a complicados argumentos que permitan delimitar con claridad el campo de acción de cada uno de ellos.

En definitiva, la deficiente técnica legislativa con la que se quiere dar respuesta punitiva a la corrupción de siempre, como si estuviéramos ante comportamientos realmente novedosos, lejos de facilitar la labor de los agentes jurídicos, se convierte en un auténtico quebradero de cabeza para muchos y, por tanto, es causante de inseguridad jurídica, un lujo que un llamado Estado de derecho no puede permitirse cuando se trata de la afectación de derechos fundamentales del ciudadano. 


\section{EL DELITO DE ENRIQUECIMIENTO ILÍCITO EN NUESTRO SISTEMA JURÍDICO PENAL}

\section{Determinación del bien jurídico protegido}

Un indicio de lo que pudiera considerarse como objeto de protección en un determinado delito lo constituye la ubicación de este en el ámbito del Código Penal (CP). Desde este punto de vista, el delito de enriquecimiento ilícito, previsto en el artículo 401 del $\mathrm{CP}$, se encuentra ubicado en la sección IV, denominada "Corrupción de funcionarios", en el seno del título correspondiente a los delitos contra la Administración Pública. Otras figuras delictivas que se encuentran en esta misma sección son, por ejemplo, el delito de cohecho en sus diversas modalidades y el tráfico de influencias, las cuales obviamente presentan un muy diferente contenido en lo que a su antijuridicidad material se refiere.

Visto así, nada impediría considerar a esta conducta como un ataque contra lo que muchos autores han definido como el "buen funcionamiento de la Administración Pública" (Gálvez Villegas, 2017). Pero decir esto es prácticamente no decir mucho acerca de lo que se quiere proteger cuando se sanciona con penas especialmente elevadas a aquel funcionario que, "abusando de su cargo", incrementa ilícitamente su patrimonio. En este particular caso, la ubicación no aporta mucho a la hora de encontrar una respuesta plenamente satisfactoria a semejante cuestión.

Por ello, resulta necesario hacer un primer acercamiento a lo que constituye el comportamiento materia de desvaloración, para poder captar de una manera más clara el sentido y la finalidad preventiva que cumple este tipo penal. De conformidad con lo dispuesto en el artículo 401 del CP, comete enriquecimiento ilícito "el funcionario o servidor público que incrementa ilícitamente su patrimonio respecto de sus ingresos legítimos" abusando de su cargo. En consecuencia, son dos los aspectos que destacan en este tipo penal: por un lado, el carácter ilícito del incremento patrimonial que presenta el funcionario (o servidor público); por otro, la idea del abuso del cargo vinculada directamente a semejante incremento.

De acuerdo con esta descripción, lo que es materia de explícita desvaloración no es, en realidad, una concreta conducta, sino un específico resultado, que se identifica con el incremento patrimonial que presenta el funcionario. El legislador ha querido abstenerse de identificar una conducta en concreto que fuera la determinante de dicho resultado; no obstante, sí ha querido destacar el hecho de que este ha de ser ilícito. Esto nos lleva necesariamente a admitir que la conducta de origen del mismo ha de tener también esa característica; es decir, ha de ser ilícita.

Muchos autores nacionales han analizado y planteado diferentes hipótesis en torno a la verdadera naturaleza de esta conducta (Rojas Vargas, 1999). Para unos, basta simplemente con que se trate de un comportamiento antijurídico, sin necesidad de que este constituya a su vez un ilícito penal; para otros, es imprescindible que semejante 
comportamiento constituya una conducta delictiva, si bien no se discrimina en torno a la naturaleza de esta; por último, hay quienes consideran que tales comportamientos delictivos han de implicar un ataque a la Administración Pública.

Este tema resulta de gran importancia si se considera que, finalmente, permite concluir que la figura del enriquecimiento ilícito tiene como presupuesto la previa ejecución de un determinado comportamiento por parte del sujeto activo; y si se viene a concluir que este ha de constituir, al menos en primera instancia, una conducta delictiva, lleva a confirmar la naturaleza subsidiaria de este tipo de injusto (Abanto Vásquez, 2003; en contra: Gálvez Villegas, 2017).

A este respecto, admitir como conducta determinante de un enriquecimiento ilícito cualquiera, siempre y cuando se ejecute al margen de la legalidad -incluyendo las simples inconductas funcionales-, implicaría una diferente desvaloración entre la conducta ejecutada por el funcionario y las consecuencias que esta le reporta, de tal manera que, no obstante aquella no es merecedora de ningún reproche en el ámbito penal, sus efectos sí. Esto genera una suerte de responsabilidad objetiva, donde el objeto de desvaloración ya no giraría en torno a la conducta, sino exclusivamente en torno al producto de la misma. En otros términos, se acabaría sancionando penalmente al funcionario no por lo que hizo, sino por lo que ello le ha reportado, lo que resulta absolutamente incompatible con la vigencia del principio de culpabilidad.

En tanto queda proscrita la responsabilidad objetiva, hay que buscar una explicación distinta a este planteamiento; ello nos lleva a considerar que el comportamiento antecedente al resultado "enriquecimiento ilícito" no puede ser más que una conducta al menos típica y antijurídica. Pero, aun así, es necesario seguir aclarando el término ilícitamente, que caracteriza el incremento patrimonial.

Es aquí donde adquiere interés el otro elemento que considerábamos esencial para un primer acercamiento a esta figura, aquel que hace referencia al abuso del cargo. Resulta indudable, por su misma sistemática, que la figura del enriquecimiento ilícito únicamente es entendible en el ámbito del ejercicio de una función pública. En primer lugar, porque, al configurarse como un delito especial, su autor solo puede tener la condición de funcionario público a los efectos penales, según lo señalado en el artículo $425 \mathrm{del}$ $\mathrm{CP}$; de esta manera, queda fuera del marco de nuestro ordenamiento jurídico la figura del enriquecimiento ilícito en particulares. En segundo lugar, porque tampoco resulta suficiente disponer de semejante condición, en la medida en que el legislador ubica a este funcionario en lo que constituye su cargo; es decir, está pensando en un funcionario que está actuando en el cumplimiento de sus labores; solo así es posible entender que pueda abusar de "su cargo" o, lo que es igual, pueda ir más allá de los deberes propios que le impone la función pública que desarrolla (Gálvez Villegas, 2017). 
Ello lleva a delimitar como fuente de la que proviene el enriquecimiento ilícito, precisamente, el ejercicio de la función pública, siendo el incumplimiento de esta la que finalmente viene a constituirse en el modus operandi del funcionario para hacerse con semejantes ganancias indebidas. De esta manera, resulta imposible entender como conducta antijurídica a partir de la cual el sujeto activo en este delito va a lograr el incremento patrimonial indebido, materia de desvaloración en el artículo 401 del CP, aquella que pudiera realizar el funcionario al margen de su cargo, por ejemplo, delitos comunes como robos, apropiaciones, tráfico de drogas, entre otros, en tanto que en estos la condición de funcionario no resulta relevante a los efectos de su tipificación, lo que determina que, por esta misma razón, al momento de su ejecución resulte absolutamente irrelevante el ejercicio o no de cargo público alguno; en tales situaciones, por tratarse de delitos comunes, el desvalor recae básicamente en la conducta que puede ser ejecutada por cualquier persona. En cambio, tratándose de delitos especiales, es la condición del sujeto activo, y cómo esta se vincula a la conducta, donde recae el grueso materia de desvaloración, como elementos inseparables.

Esta circunstancia es la que prima en el ámbito del delito de enriquecimiento ilícito, donde lo verdaderamente relevante no es solo el que se desvalore el resultado "enriquecimiento ilícito", sino el que este tenga una vinculación directa con el ejercicio de la función pública, siendo ello lo que justifica su misma existencia.

De esta manera, puede llegar a perfilarse el contenido del bien jurídico protegido en este delito. En tanto el desvalor no recae en un incumplimiento concreto de algún deber especial por parte del funcionario público -tal y como ocurre con otras figuras delictivas como el cohecho, la colusión o el peculado, donde puede entenderse que el objeto de protección gira en torno a ese cumplimiento particular que le corresponde al funcionario, dependiendo de las responsabilidades que le atribuye su cargo-, en el ámbito del enriquecimiento ilícito la materia de desvaloración gira en torno a los efectos lucrativos que para el funcionario le reporta un genérico incumplimiento de sus funciones, mientras este no se encuentre subsumido en otro tipo penal específico.

Así, se garantiza el uso legítimo de la función pública como fuente generadora de beneficios económicos lícitos, es decir, como fuente legítima de ingresos; de ahí que, cuando esta es utilizada como una forma de lucro personal indebido, sin que sea susceptible de subsunción bajo un tipo penal concreto, será admisible el recurso al enriquecimiento ilícito. Ello determina su clara naturaleza subsidiaria.

Un argumento que contribuye a apoyar este planteamiento se deriva de la ubicación sistemática de esta figura en el marco de los llamados delitos contra la Administración Pública, cometidos por funcionarios. El delito de enriquecimiento ilícito se encuentra en el último de los preceptos, incluso por detrás de otra figura también ciertamente extraña en cuanto a la definición de su objeto de protección, como es el delito de tráfico 
de influencias. Si aquí se considera que el objeto de protección es el prestigio de la Administración, cuando este es usado como mecanismo para obtener un beneficio indebido (Muñoz Conde, 2017); en el enriquecimiento indebido, es precisamente materia de desvaloración el uso de la función pública como mecanismo de lucro personal indebido, siguiendo de esta manera una misma línea de comportamiento.

\section{Algunos aspectos relevantes de la tipicidad objetiva}

Configurándose como un delito especial, el sujeto activo no puede ser nadie más que un funcionario público, entendido dicho término como especifica el artículo 425 del CP; por otro lado, la condición de funcionario tampoco resulta suficiente, en la medida en que el elemento que es materia de desvaloración, esto es, el enriquecimiento indebido, encuentra su origen en el cargo que este desempeña, de ahí que ha de ser un funcionario que hace uso de las prerrogativas que su cargo le confiere como mecanismo para la obtención de un lucro que no guarda relación con la remuneración que le corresponde, de conformidad con el simple ejercicio de su cargo.

Ello implica que el mecanismo empleado por el funcionario para lograr ese enriquecimiento es el ejercicio de su propio cargo, de tal forma que si, por ejemplo, la secretaria del ministro obtiene una jugosa cantidad de dinero gracias a que logró que este firmara un importante contrato con una determinada empresa, en realidad, estaríamos hablando de un eventual tráfico de influencias más que de un acto de enriquecimiento ilícito, por cuanto su conducta nada tiene que ver con las obligaciones propias de su cargo. De esta manera queda excluida - como ya indicamos con anterioridad-la posibilidad de admitir el enriquecimiento ilícito de particulares, al que se equiparan también quienes en el pasado tuvieron la condición de funcionarios, pero obtienen provecho económico de dicha situación, tal y como sucede con aquellos ex altos cargos que, por sus "contactos" logrados en el ejercicio de su función, pueden seguir sacando provecho de ello. Esto nos llevaría, en todo caso, a la figura de tráfico de influencias, según el artículo 400 del CP.

Siendo delito especial, no hay en la actualidad ninguna duda sobre la participación de un extraneus en su comisión, aplicándose así las reglas generales ya conocidas. Esta circunstancia ha sido ya incluso admitida sin problemas por parte de la jurisprudencia (Acuerdo Plenario 3-2016/CJ-116); pero muchos de los casos que se vienen planteando como presuntos supuestos de participación caen en un mismo error de tipificación: en realidad, no son actos de una eventual complicidad en el delito de enriquecimiento, sino auténticos supuestos de otra figura delictiva, la de lavado de activos.

Así, allí donde se hace uso de testaferros o de terceras personas que tienen como finalidad básica el evitar vincular al funcionario público con sus ingresos indebidamente obtenidos, se está procediendo con comportamientos que nada tienen que ver con la misma obtención de estos, que es la conducta tipificada como enriquecimiento ilícito; 
buscan evitar que sea descubierto el desbalance patrimonial que presenta el funcionario, dando una falsa apariencia de titularidad respecto a un patrimonio que no es tal. La complicidad en el delito de enriquecimiento ilícito debe implicar un acto de colaboración con el funcionario en la obtención indebida del beneficio económico; así, por ejemplo, intermediar en el cobro de dinero a favor del funcionario o incluso participar de las mismas negociaciones en la determinación del lucro que se obtendrá en nombre de este. Pero si la intervención tiene lugar luego de que ya fue otorgado el beneficio, definitivamente, la consumación de esta conducta ya se produjo y los actos posteriores a ella, por definición, no pueden calificarse como actos de ayuda en la ejecución de este delito, sino que se convierten en auténticos actos que buscan justificar mediante terceros la legitimidad de un patrimonio que no encuentra sustento en las remuneraciones propias del puesto ocupado en el ámbito de la Administración Pública.

Entendemos que no hay incompatibilidad alguna entre el delito de enriquecimiento ilícito y el de lavado de activos, por cuanto el primero puede presentarse también como delito fuente que da pie a la comisión del segundo. A este respecto, no obstante, vía acuerdo plenario, se ha querido diferenciar entre actos de colaboración en el delito de enriquecimiento ilícito y el lavado de activos, en función de que la intervención del extraneus fuera coetánea al ejercicio del cargo, dándose a entender que ambas figuras no pueden concurrir en el tiempo.

El agente, por tanto, debe realizar mientras ostenta su condición funcionarial una secuencia de actos provenientes de la misma resolución criminal (producir enriquecimiento ilícito); pero, además, la mejora acumulativa de su patrimonio que va obteniendo debe él mantenerla hasta el fin del periodo que ejerce su cargo funcional, sea este por cese o destitución. Lo cual posibilita que la intervención del tercero extraneus pueda ocurrir durante todo el tiempo que el funcionario intraneus se mantenga en el ejercicio de su posición y competencia funcionarial. Sea, pues, como instigador cuando motive o induzca a la realización de actos idóneos para el enriquecimiento; o como cómplice cuando ayude, de cualquier manera, a obtener, recepcionar, administrar, guardar, transferir o mantener los ingresos, los bienes, créditos o réditos que van produciendo el enriquecimiento ilícito del intraneus, el tercero deberá siempre de intervenir mientras el mal funcionario conserve su cargo y condición. Por consiguiente, si la conducta del tercero se realiza o tiene lugar con posterioridad al cese o después de la pérdida del estatus funcionarial del agente del enriquecimiento ilícito, esto es, después que concluya la continuidad de la actividad delictuosa y cese la permanencia, la conducta que despliegue aquel ya no será accesoria, sino autónoma y deberá ser considerada como un delito diferente que bien puede ser el de lavado de activos. (Acuerdo Plenario 3-2016/CJ-116)

Mucho más complejo resulta el análisis de la conducta delictiva materia de criminalización en este tipo penal, debido a que básicamente no se describe ninguna. Cuando el legislador tipifica este delito, hace expresa mención a la idea de "incrementar ilícitamente el patrimonio", pero sin especificar cómo ha de producirse dicho resultado. Antes 
indicábamos que, en realidad, en esta figura lo que se desvalora es un concreto resultado, mas no el origen del mismo, lo que sin duda constituye uno de los problemas más importantes de cara a la correcta delimitación típica de la misma.

No obstante, también anticipábamos que un primer criterio delimitador lo obtenemos a partir de la expresión "abusando de su cargo", empleada por el legislador como elemento con el que está directamente relacionado el resultado del incremento patrimonial ilícito que presenta el funcionario público. A este respecto, la idea de abuso vinculada al cargo ejercido por el sujeto activo de este delito constituye un elemento esencial que ayuda a su mejor entendimiento. En primer lugar, porque permite afirmar que la condición de funcionario no es suficiente, sino que ha de tratarse necesariamente de un funcionario que actúa en el ámbito de sus competencias. En segundo lugar, porque al desvalorarse las consecuencias lucrativas que representa para este el ejercicio del cargo, más allá de la remuneración que conlleva el ejercicio del mismo, permite considerar que allí donde sea materia de desvaloración penal la infracción de un concreto deber en el cumplimiento del cargo, será esta figura la que corresponda aplicar, por exigencias del principio de tipicidad. De esta manera, el delito de enriquecimiento ilícito queda como una figura de aplicación subsidiaria.

Si el incremento patrimonial que presentara el funcionario tuviera su origen, por ejemplo, en las dádivas que este ha venido obteniendo a lo largo del tiempo en el que se ha desempeñado en su cargo, ya sea que haya o no incumplido de manera expresa sus obligaciones, no cabe la menor duda de que ello comporta una conducta calificable bajo la figura de cohecho pasivo, ya sea propio o impropio, que repercute, económicamente hablando, en un incremento indebido de su patrimonio. Pero lo que es materia de desvaloración bajo dicha calificación jurídica es específicamente la solicitud de tal dádiva a la que se vincula directamente el ejercicio de la función pública, sin que interesen las consecuencias económicas que ello le reporte al funcionario. Y esto se entiende si partimos de que el objeto de protección en dicha figura delictiva es el deber de imparcialidad con el que ha de actuar la Administración Pública; el beneficio económico que ello le genera al funcionario solo puede entenderse como un acto de agotamiento del delito, carente, en consecuencia, de cualquier repercusión penal.

Si el abuso de función no puede subsumirse bajo la tipificación de cualquier otra conducta, es entonces cuando hay que advertir si este ha contribuido al incremento del patrimonio del funcionario, porque solo así podrá considerarse este "indebido" y calificarse como enriquecimiento ilícito. En ello estriba la naturaleza subsidiaria de este delito, de ahí que no sea posible admitir un eventual concurso delictivo entre este delito y aquellos que desvaloran de manera específica determinados incumplimientos de función. Sería imposible admitir bajo esta lógica, por ejemplo, un concurso entre un delito de cohecho y un delito de enriquecimiento ilícito, ya que el primero se configura como la fuente de la que el funcionario obtiene su indebido beneficio y la misma descripción 
típica del cohecho conlleva ya una desvaloración implícita al beneficio que le reporta al funcionario la obtención de la ventaja a cambio de una concreta actuación en su cargo. Lo mismo tendría lugar si se tratara de un eventual delito de peculado.

Es en realidad un problema de interpretación de normas más que de concurso de delitos. El delito de enriquecimiento ilícito se presenta en el artículo 401 del CP como un tipo penal genérico y particularmente abierto, lo que lo convierte en una norma con un ámbito de aplicación extraordinariamente restringido.

Esta característica peculiar se convierte, a su vez, en uno de los principales puntos de crítica a esta figura, e incluso es uno de los factores que determinan que ciertas legislaciones se resistan a su inclusión en el Código Penal (Abanto Vásquez, 2003). Solo podrá imputarse este delito en el caso de que no se establezca con certeza la procedencia del enriquecimiento del funcionario, pero a su vez ello obliga de todas maneras a definir el origen ilícito del mismo, lo que no puede encontrar un origen distinto al del abuso del cargo. Es decir, si un funcionario presentara un evidente desequilibrio patrimonial no justificable en sus ingresos declarados, sin que, por otro lado, haya indicios de comisión de otros delitos relacionados con el cargo, ¿sería esto suficiente para admitir ya la presencia de un delito de enriquecimiento ilícito? La respuesta a esta cuestión ha de ser contundentemente no, y ello por exigencias del principio de tipicidad, así como por respeto al principio de presunción de inocencia: el eventual desbalance patrimonial que pueda presentar un funcionario público puede ser un indicio suficiente para, a partir de ahí, justificar el inicio de una investigación sobre el origen de dicho desbalance, pero en absoluto puede ser suficiente para sustentar una eventual responsabilidad por enriquecimiento ilícito.

Este problema pretende encontrar un atisbo de solución legal a partir del 2001, luego de la modificación que se realizara al artículo 401 del CP donde se termina por identificar como indicio de enriquecimiento ilícito el carácter notoriamente superior del aumento del patrimonio o del gasto económico personal del funcionario respecto al que haya presentado en virtud de sus ingresos lícitamente percibidos. Se pretende así superar los cuestionamientos que pudieran plantearse sobre un tipo penal tan absolutamente abierto como el que se prevé, al sancionarse con penas de hasta diez años al funcionario que "por razón de su cargo" se enriqueciera ilícitamente. El carácter indiciario del simple desbalance patrimonial permitiría así circunscribir, en una primera instancia, el origen del mismo a comportamientos relacionados con la función pública, pero, a partir de ahí, obliga al menos a identificar a qué clase de conductas realizadas en el ámbito del cargo obedece semejante resultado económico presentado por las finanzas del funcionario en cuestión. De esa manera, si en el curso de dicha investigación llegara a determinarse que la obtención de ese lucro ha sido posible a partir de comportamientos que encuentran ya una concreta tipificación bajo otras figuras delictivas, definitivamente serían estas las aplicables en detrimento de la de enriquecimiento ilícito. 


\section{ALGUNAS CONSIDERACIONES EN TORNO AL DELITO DE LAVADO DE ACTIVOS}

No toca aquí hacer un análisis de esta figura delictiva, porque ello supondría exceder los límites permitidos para el presente trabajo. Por esta razón vamos a ser selectivos en el puntual estudio de aquellos aspectos que son los que pueden servirnos de clave para poder responder lo que constituye nuestra pregunta de fondo, que es determinar la relación existente entre este delito y el de enriquecimiento ilícito.

Debemos comenzar fijando cuál es la finalidad que, desde un punto de vista de política criminal, justifica la criminalización de lo que hoy se concibe como diversas modalidades del delito de lavado de activos contenidas en el Decreto Legislativo 1106, "Decreto Legislativo de lucha eficaz contra el lavado de activos y otros delitos relacionados a la minería ilegal y crimen organizado", modificado mediante el Decreto Legislativo 1367 , del 29 de julio del 2018. Es evidente que ello va de la mano de la concepción del bien jurídico protegido en este delito. En este sentido, y más allá de que ya a nivel nacional haya un cierto consenso en la caracterización de este delito como pluriofensivo (Acuerdo Plenario 03-2010/CJ-116), lo que resulta evidente es que con ello se busca implementar un medio eficaz en la lucha contra cierta clase de criminalidad caracterizada por la obtención de elevados niveles de beneficio económico, que acaba utilizando las múltiples alternativas de inversión que ofrece el sistema económico financiero de un Estado para poder disfrutar, en el ámbito de lo lícito, de los réditos lucrativos que generan semejantes actividades delictivas.

Ello determina que hablar de lavado de activos no es posible si no es a partir de la previa comisión de una determinada actividad delictiva que se convierte en esa fuente generadora de beneficios económicos indebidos, que más tarde serán materia de ese "maquillaje" que representa el lavado de activos, a través del cual se les quiere dar un aparente origen lícito. Es a lo que la doctrina y la jurisprudencia denominan delito fuente (Pariona Pastrana, 2017); su explícita referencia legal se encuentra en el artículo 10 del antes señalado Decreto Legislativo 1106. Lo interesante de su mención legal, más allá de la referencia a ciertas figuras delictivas, es que finalmente se hace alusión a "cualquier otro (delito) con capacidad de generar ganancias ilegales", lo que ayuda a delimitar el ámbito de aplicación del lavado de activos, que queda de esta manera vinculado solo a cierta clase de actividades criminales generadoras de lucro, entendido este como un incremento indebido del patrimonio.

En consecuencia, esto permite considerar como delitos fuente, en el marco de actos de lavado de activos, solo a ciertas figuras delictivas dentro del grupo de los delitos contra la Administración Pública, en las cuales ese elemento del beneficio económico esté presente. Pueden mencionarse los delitos de peculado, cohecho pasivo, tráfico de influencias y, por qué no, el de enriquecimiento ilícito. De una u otra manera, en las muy diferentes estructuras típicas propias de los mencionados actos delictivos, está 
presente la idea del beneficio patrimonial. En cambio, el delito de abuso de autoridad o el delito de colusión no podrían configurarse como delitos fuente en el ámbito del lavado de activos, dado que, según su misma estructura típica, en ellos no está presente ese elemento generador de ganancias ilegales que se exige en el ya mencionado artículo 10 del Decreto Legislativo 1106.

Así pues, en el ámbito teórico, el delito de enriquecimiento ilícito podría configurarse como un delito fuente respecto a un acto posterior de lavado de activos, ya que gira en torno a la obtención de un incremento patrimonial a partir de un comportamiento indebido consistente en el abuso de las funciones públicas ejercidas por el funcionario público. Por lo tanto, hay un explícito componente económico como parte de su definición típica, que es precisamente el que caracteriza el delito fuente como presupuesto indispensable para el acto de lavado de activos.

Sin embargo, esa apreciación queda en el ámbito teórico porque en el ámbito práctico es donde realmente se presentan las dificultades a causa de la naturaleza subsidiaria del delito de enriquecimiento indebido: si ese beneficio económico obtenido por el funcionario encuentra su origen en conductas expresamente tipificadas, siendo parte de la desvaloración que conlleva ese específico tipo de injusto, no tiene cabida alguna la figura del enriquecimiento indebido; en ese caso, es aplicable el tipo penal concreto. Por ejemplo, si el funcionario se ha dedicado a cometer sucesivos actos de corrupción que le han reportado cantidades de dinero que oculta en diferentes cuentas bancarias a nombre de terceras personas, el delito fuente en este caso encuentra su específica definición en el delito de cohecho pasivo que ha cometido el sujeto, dado que dicha conducta es la que directamente ha generado semejantes ganancias ilícitas, cuyo ocultamiento posterior es calificado como el acto de lavado de activos.

La indefinición de la fuente generadora del incremento patrimonial no es suficiente para subsumirla bajo la calificación del artículo 401 del CP. Se requiere la presencia de dos componentes: uno de naturaleza positiva, como es el incremento patrimonial que presente el funcionario público; y otro de naturaleza negativa, obtenido a partir del abuso de sus funciones no calificable bajo ninguno de los tipos penales ya previstos en el ámbito de los delitos contra la Administración Pública cometidos por funcionarios públicos. Estas circunstancias hacen poco viable su aplicación efectiva, y es aún más complicado que pueda configurarse como delito fuente en el ámbito del lavado de activos.

Lo que acabamos de señalar parte de una idea previa: los delitos de enriquecimiento ilícito y de lavado de activos no pueden concurrir en una misma conducta; es decir, son figuras incompatibles entre sí. Ello se debe básicamente a que su estructura típica parte de comportamientos muy diversos, los cuales reflejan, en el fondo, la diferente última ratio que justifica su existencia en el ámbito de nuestro ordenamiento jurídico. 
Así, por ejemplo, cuando el funcionario presente propiedades a su nombre que no pueden justificarse a partir de los honorarios que percibe por razón de su cargo, semejante situación sería amparable bajo una previa calificación de enriquecimiento ilícito, mas no de lavado de activos, por cuanto a través de esta figura lo que expresamente se pretende sancionar es el aprovechamiento indebido de los mecanismos legales que ofrece el mercado de bienes y capitales para dotar de apariencia de legalidad a lo que proviene de una previa actividad ilícita. Por tanto, la mera titularidad de un patrimonio no ajustado a las ganancias declaradas por el funcionario no constituye acto alguno de lavado de activos, dado que en semejante supuesto ese desfase económico es manifiesto, mas no sustentable patrimonialmente, idea que es la que justifica el desvalor característico del delito de enriquecimiento ilícito.

No obstante, no podemos desconocer el hecho de que tales situaciones no son las más comunes: en la práctica, el desbalance patrimonial se procede a ocultar a partir del recurso a testaferros o al uso de sociedades opacas, donde finalmente resulta muy difícil identificar al verdadero titular. No cabe duda de que en semejantes contextos, incluso hasta desde un punto de vista temporal, se suceden diversas acciones en el tiempo que nos van a permitir identificar y calificar las distintas conductas delictivas que acaban relacionándose entre sí, no en virtud de un eventual concurso, sino que acaban configurándose en figuras conexas entre sí, donde una va a ser posible solo y exclusivamente porque previamente se ejecutó la otra. Volvemos así a la definición del delito fuente en el ámbito de la estructura del delito de lavado de activos.

En situaciones como las descritas, puede perfectamente diferenciarse dos comportamientos. En primer lugar, el que permite la obtención del patrimonio indebido y que facilita disponer de este, lo que definiría un eventual acto de enriquecimiento ilícito. En segundo lugar, y que se sucede en el tiempo, aquel que permite su transformación en patrimonio lícito, o que pretende desviar el conocimiento de su origen ilícito, el cual constituye sin más un comportamiento típico de lavado de activos.

\section{CONCLUSIONES}

1. En el análisis que se ha realizado en torno a la figura del delito de enriquecimiento ilícito, se han puesto de manifiesto las múltiples dificultades que, tanto desde el punto de vista material como desde el punto de vista formal, se plantean de cara a su efectiva aplicación. Ya no hay duda alguna de que la presencia de esta conducta en el marco de los delitos cometidos por funcionarios públicos pretendía criminalizar la mera tenencia de un patrimonio no justificado, ante la imposibilidad de poder descubrir la fuente ilícita generadora del mismo. Sin embargo, su misma descripción típica, así como su relación con el resto de comportamientos que aparecen contenidos bajo esa denominación de delitos 
cometidos por funcionarios públicos, son las principales trabas que imposibilitan una efectiva aplicación de este precepto que termina por ser relegado a favor de la aplicación de otros tipos penales. Se reduce así a una simple intención de principios carente de toda eficacia preventiva.

2. A ello también contribuye el que una aplicación del artículo 401 del CP respetuosa del principio de legalidad, en directa vinculación con el de presunción de inocencia, impide calificar como enriquecimiento ilícito y, por consiguiente, condenar por semejante acto delictivo el mero hecho de contar, siendo funcionario público, con un desbalance patrimonial que no encuentra su justificación en las retribuciones declaradas; ello, en todo caso, podría ameritar una eventual investigación fiscal. La necesidad de vincular ese desbalance patrimonial a actos funcionales irregulares se convierte en el eje central de esta figura, lo que exige acreditar y definir la fuente de la que procede ese patrimonio indebido. Si ello conduce a actos ya tipificados bajo específicas formas delictivas, la figura del enriquecimiento ilícito, sencillamente, cede su paso ante esa particular conducta criminal generadora del incremento patrimonial ilícito.

3. El aparente conflicto entre el enriquecimiento ilícito y el delito de lavado de activos es de naturaleza sustantiva, y se soluciona a partir de una adecuada identificación de comportamientos que permita subsumir bajo cada uno de estos tipos penales aquellas conductas que confluyen de una manera aparente. A este respecto, no debe perderse de vista que, tanto por su estructura como por el contenido de la antijuridicidad material propia de cada uno de ellos, no pueden confluir en un aparente concurso de delitos, de ahí que no haya ningún inconveniente en admitir la calificación del delito de enriquecimiento ilícito como posible delito fuente respecto a un delito de lavado de activos, si bien la principal dificultad que dicha afirmación va a plantear en la práctica deriva de las mismas dificultades típicas que complican su aplicación, tal y como hemos puesto de manifiesto a lo largo del presente trabajo.

4. En definitiva, y de cara a una política criminal de lege ferenda, quizá sería recomendable repensar la existencia de figuras delictivas como la del enriquecimiento ilícito que -creadas más para aligerar problemas de prueba en la práctica ante eventuales investigaciones fiscales, que en aras de garantizar un bien jurídico en concreto- no han contribuido en nada a la lucha contra la criminalidad en el ámbito de la Administración Pública. A su vez, esta figura ha fomentado confusión con otras figuras, como el lavado de activos que, en función del respeto que se merece el principio de legalidad, ha de ser aplicada con un mayor rigor técnico que deje de entender dicha figura como la mera criminalización de la sospecha ante la falta de pruebas concretas sobre el origen ilícito de los bienes materia de blanqueo. 


\section{REFERENCIAS}

Abanto Vásquez, M. (2003). Los delitos contra la Administración Pública en el Código Penal peruano. Palestra Editores.

Gálvez Villegas, T. A. (2017). El delito de enriquecimiento ilícito. Instituto Pacífico.

Muñoz Conde, F. (2017). Derecho penal. Parte especial. Tirant lo Blanch.

Pariona Pastrana, J. (2017). El delito precedente en el delito de lavado de activos. Instituto Pacífico.

Rojas Vargas, F. (1999). Delitos contra la Administración Pública. Grijley.

X Pleno Jurisdiccional de las Salas Penales Permanente y Transitoria. Acuerdo Plenario 3-2016/CJ-116 [Corte Suprema de Justicia]. 12 de junio del 2017. file:///C:/Users/ User/Downloads/PRECEDENTES-2017-I-58-77.pdf 